\title{
Cerebral and peripheral muscle oxygenation and perfusion: Course in moderate and late preterm neonates during the first day after birth
}

\author{
N. HOELLER ${ }^{1,2}$, N. BAIK-SCHNEDITZ ${ }^{1,2}$, B. SCHWABERGER ${ }^{1,2}$, \\ L. MILEDER ${ }^{1,2}$, B. URLESBERGER ${ }^{1,2}$ and G. PICHLER ${ }^{1,2 *}$
}

\begin{abstract}
${ }^{1}$ Research Unit for Neonatal Micro- and Macrocirculation, Department of Paediatrics and Adolescent Medicine, Medical University of Graz, Graz, Austria

${ }^{2}$ Division of Neonatology, Department of Paediatrics and Adolescent Medicine, Medical University of Graz, Graz, Austria
\end{abstract}

Received: October 17, 2019 • Accepted: May 07, 2020

Published online: July 17, 2020

(C) 2020 Akadémiai Kiadó

\section{ABSTRACT}

Aim: To investigate the ratio of cerebral tissue oxygenation index (cTOI) to peripheral muscle tissue oxygenation index (pTOI) measured by near-infrared spectroscopy (NIRS) in cardio-circulatory stable preterm neonates without signs of inflammation/infection on the first day after birth. Methods: Observational study analysing secondary outcome parameters of the 'Avoiding Hypotension in Preterm Neonates (AHIP)' trial (ClinicalTrials.gov identifier: NCT01910467). Preterm neonates, who had cTOI and pTOI measurements during $24 \mathrm{~h}$ after birth, were included. In each neonate the mean of the cTOI/ pTOI-ratio, cTOI, pTOI and routine monitoring parameters were calculated for each hour and for the 24-h measuring period. Courses of all measured parameters were analysed. Results: Eighty-seven stable preterm neonates (33.1 [32.1-34.1] weeks of gestation) were included. The mean value over the 24-h measuring period for the cTOI/pTOI-ratio was $0.96 \pm 0.02$, for cTOI $70.1 \pm 1.4$ and for pTOI $73.4 \pm 0.9$. Routine monitoring parameters were in the normal ranges over $24 \mathrm{~h}$. The courses of the cTOI/pTOIratio and cTOI showed significantly lower values from hour 5 to 15 compared to the first hours after birth. Heart rate decreased significantly over time, whereas mean arterial blood pressure increased significantly. pTOI, arterial oxygen saturation and body temperature showed no significant change over time. Conclusion: We are the first to report on cTOI/pTOI-ratios for cardio-circulatory stable preterm

\footnotetext{
* Corresponding author. Division of Neonatology, Department of Paediatrics and Adolescent Medicine, Medical University of Graz, Auenbruggerplatz 30, 8036 Graz, Austria. Tel.: +43 316385 80520; fax: +43 316385 13300, E-mail: gerhard.pichler@medunigraz.at
} 
neonates over a 24-h period after birth, showing significantly lower values from hour 5 to 15 compared to the first hours after birth.

\section{KEYWORDS}

cerebral oxygenation, near-infrared spectroscopy, peripheral muscle oxygenation, preterm neonates, haemodynamic changes

\section{INTRODUCTION}

Near-infrared spectroscopy (NIRS) measurements of cerebral and peripheral tissue oxygenation in critically ill neonates for detection of early stages of shock are of increasing clinical interest. Early cardio-circulatory signs of inflammation, especially in case of developing sepsis, are difficult to be recognized in neonates. In sepsis, microvascular dysfunction occurs secondary to perfusion heterogeneity, arterio-venous shunting and impaired autoregulation [1, 2]. A correlation between microcirculatory abnormalities and organ dysfunction has already been demonstrated in adults using NIRS [3]. However, routine monitoring parameters may fail to detect early microcirculatory dysfunction [4].

Most clinical studies in term and preterm neonates on cerebral oxygenation used "continuous wave spatially resolved technique" NIRS [5-11]. There are only few studies of simultaneous measurement of cerebral and peripheral muscle oxygenation and perfusion in term and preterm neonates. Grossauer et al. [12] compared simultaneously measured cerebral tissue oxygenation index (cTOI) and peripheral muscle tissue oxygenation index (pTOI) and described a cTOI/pTOI-ratio of $1.14 \pm 0.14$ for healthy preterm and term neonates within the first eight weeks of life. Regarding regional cerebral oxygenation and regional peripheral muscle oxygenation during the immediate postnatal transition, cerebral tissue oxygenation reaches a plateau faster compared to peripheral muscle oxygenation $[13,14]$.

On the one hand, Pichler et al. [15] described a decrease of pTOI in healthy term neonates from $67 \%$ to $61 \%$ during the first week of life. On the other hand, Naulaers et al. described an increase of cTOI in premature infants over the first three days of life from 57\% to 76.1\% [16].

In a single-centre randomised controlled study entitled 'Avoiding Hypotension in Preterm Neonates (AHIP; ClinicalTrials.gov identifier: NCT01910467), Pichler et al. [17] used simultaneous cerebral and peripheral muscle NIRS measurements in combination with dedicated intervention guidelines to help avoiding arterial hypotension and catecholamine administration in preterm neonates, resulting in a non-significant reduction in burden of arterial hypotension.

The aim of the present study was to investigate simultaneously measured cTOI and pTOI using NIRS in cardio-circulatory stable preterm neonates without signs of inflammation/ infection on the first day after birth, and to analyse the course of the cTOI/pTOI-ratio during this period for the first time. Based on the findings of Pichler et al. [15] and Naulaers et al. [16] we hypothesized that the cTOI/pTOI-ratio increases during the first day after birth.

\section{METHODS}

\section{Patients}

In the present observational study, which was part of the AHIP trial [17] performed at the neonatal intensive care unit (NICU), Medical University of Graz, Austria, secondary outcome 
parameters were analysed. Preterm neonates of less than $37+0$ weeks of gestation born from October 2013 to December 2016 with risk for inflammation/infection after premature rupture of membranes, amnion infection syndrome or increased markers of maternal systemic inflammation (C-reactive protein [CRP]/leucocyte counts) were considered for inclusion in the AHIP trial. Further inclusion criteria were: decision to conduct full life support, parental written informed consent, postnatal age under six hours and no administration of catecholamines before initiation of NIRS measurements. Neonates with congenital malformations were excluded. The study was approved by the Regional Committee on Biomedical Research Ethics of the Medical University of Graz (EK number: 25-237 ex 12/13).

For the present study we included only preterm neonates without signs of infection/ inflammation. This was defined as umbilical cord Interleukin-6 $<100 \mathrm{pg} / \mathrm{mL}$ [18] and/or negative blood culture from umbilical cord blood and/or leucocyte counts $<34,000 / \mu \mathrm{L}$ [19] and/ or CRP $<10 \mathrm{mg} / \mathrm{L}[20,21]$ on the first or second day after birth.

The medical history of any pathologic findings during pregnancy and birth was collected. Furthermore, demographic and perinatal data, respiratory support and main diagnoses were documented for each neonate.

Cerebral ultrasound was performed in each neonate at the beginning and the end of NIRS measurements, as well as on postnatal days 4, 7, 14 and before discharge. Clinical follow-up was conducted until term age or until discharge, whatever came first.

Any adverse reactions during measurements were documented.

\section{NIRS measurements}

NIRS measurements were started within the first six hours of life and lasted for $24 \mathrm{~h}(24-30 \mathrm{~h}$ after birth); they were performed with a NIRO-200NX device (Hamamatsu Photonics, Hamamatsu City, Japan). The cerebral optodes were placed over the left frontal head; the interoptode distance was $4.0 \mathrm{~cm}$. The peripheral muscle optodes were placed over the right forearm; the interoptode distance was $3.0 \mathrm{~cm}$ in neonates $>1,500 \mathrm{~g}$ and $2.0 \mathrm{~cm}$ in neonates $<1,500 \mathrm{~g}$. The cerebral NIRS optodes were repositioned at least every six hours to prevent skin lesions.

Cerebral and peripheral muscle oxygenation was measured continuously and displayed as TOI. The sample interval was $2 / \mathrm{s}$.

\section{Monitoring of vital parameters}

For the present study routine monitoring data (heart rate $[\mathrm{HR}]$, pulse oximetry, mean arterial blood pressure [MABP], peripheral and central body temperature) were analysed during NIRS monitoring. Preductal arterial oxygen saturation $\left(\mathrm{SpO}_{2}\right), \mathrm{HR}$ and MABP were measured using the IntelliVue MP50 monitor (Philips, The Netherlands). Data were stored in a polygraphic system (alpha-trace digital MM, B.E.S.T. Medical Systems, Vienna, Austria) and analysed. The pulse oximetry sensor was applied on the right palm or wrist for monitoring of $\mathrm{SpO}_{2}$ and $\mathrm{HR}$. If neonates had an intra-arterial line due to medical reasons, continuous invasive blood pressure measurements were performed. In case of non-invasive blood pressure measurements the pneumatic cuff was placed around the left upper arm. These measurements were performed at least every $30 \mathrm{~min}$. Furthermore, a rectal and a skin sensor were used for central and peripheral temperature measurements. 


\section{Statistical analysis}

In each neonate mean values of cTOI, pTOI, $\mathrm{HR}, \mathrm{SpO}_{2}, \mathrm{MABP}$, as well as rectal and peripheral temperature were calculated for every hour. Mean values were calculated from the data recorded during an hour, whereby in all neonates at least in each hour data of half an hour were available. cTOI and pTOI values higher than the simultaneously measured $\mathrm{SpO}_{2}$ were excluded. Furthermore, values, which were extremely out of the range, compared to values at the hours before and afterwards were also excluded. These values were supposed to be artefacts due to loose optical contact of the sensors. Out of the mean values of cTOI and pTOI for each hour, cTOI/pTOI-ratios were calculated.

Furthermore, mean 24-h values of cTOI, pTOI, cTOI/pTOI-ratio, HR, $\mathrm{SpO}_{2}, \mathrm{MABP}$, rectal and peripheral temperature were calculated.

Mixed model ANOVA was used to assess the course of cTOI, pTOI, cTOI/pTOI-ratio, HR, $\mathrm{SpO}_{2}, \mathrm{MABP}$, rectal and peripheral temperature over the 24 -h measuring period compared to the first hour of measurements.

Data are presented as mean and standard deviation (SD) for normally distributed continuous variables and median plus 25th to 75th percentile if the distribution was skewed.

The statistical analyses were performed using SPSS Statistics 25 (IBM Corporation; Armonk, New York, USA).

\section{RESULTS}

A total number of 108 preterm neonates with less than $37+0$ weeks of gestation and at risk of inflammation/infection were measured. Ten $(9.3 \%)$ of these preterm neonates had to be

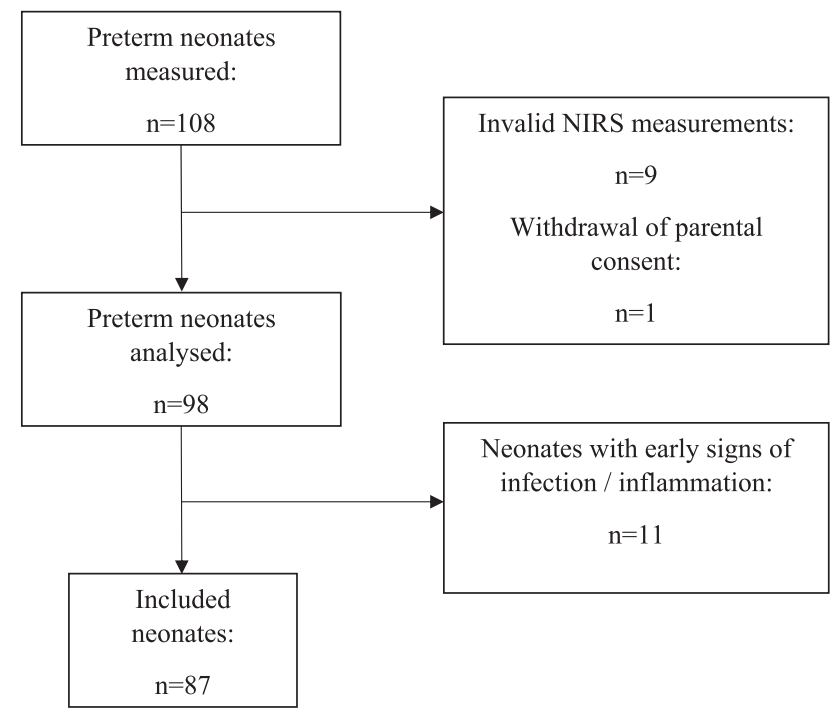

Fig. 1. Flow diagram demonstrating the number of included neonates and rationales for exclusion 
Table 1. Demographic and clinical parameters of preterm neonates. Continuous data are presented as mean $\pm \mathrm{SD}$ or median (25th percentile to 75 th percentile) based on distribution

Patients $(n)$

Females/males $(n)$

Caesarean section/vaginal delivery $(n)$

Gestational age (weeks)

Postnatal age at initiation of NIRS measurements (h)

Birth weight (g)

Umbilical artery $\mathrm{pH}$

Umbilical venous $\mathrm{pH}$

Apgar 1 minute

Apgar 5 minutes

Apgar 10 minutes

Diameter of the upper arm $(\mathrm{cm})$

Thickness of subcutaneous fat on the upper arm, measured by ultrasound (cm)

Circumference of the upper arm $(\mathrm{cm})$

Central capillary refill time at the beginning of measurement (s)

Central capillary refill time at the end of measurement (s)

Peripheral capillary refill time at the beginning of measurement (s)

Peripheral capillary refill time at the end of measurement (s)
$87 / 108(88.8 \%)$

$44(50.6 \%) / 43(49.4 \%)$

$65(74.7 \%) / 22(25.3 \%)$

$33.1(32.1-34.1)$

$2.5(1.5-4.0)$

$1859.9 \pm 488.0$

$7.30 \pm 0.05$

$7.35 \pm 0.06$

8 (8-9)

$9(8-10)$

$9(9-10)$

$2.4 \pm 0.4$

$0.3 \pm 0.1$

$8.1 \pm 1.2$

$2.7 \pm 0.6$

$2.7 \pm 0.7$

$2.6 \pm 0.6$

$2.6 \pm 0.6$

Table 2. Mean NIRS parameters of preterm neonates during the 24-h measuring period. Data are presented as mean $\pm \mathrm{SD}$

\begin{tabular}{lccc}
\hline Hour & cTOI/pTOI-ratios & cTOI $(\%)$ & pTOI $(\%)$ \\
\hline 1 & $1.00 \pm 0.15$ & $73.1 \pm 9.1$ & $73.3 \pm 7.4$ \\
2 & $1.00 \pm 0.14$ & $73.0 \pm 9.5$ & $73.8 \pm 6.7$ \\
3 & $0.97 \pm 0.17$ & $71.4 \pm 10.4$ & $74.2 \pm 7.1$ \\
4 & $0.97 \pm 0.17$ & $71.0 \pm 10.5$ & $73.8 \pm 6.8$ \\
5 & $0.92 \pm 0.14$ & $68.7 \pm 9.4$ & $75.4 \pm 5.3$ \\
6 & $0.95 \pm 0.16$ & $70.5 \pm 10.2$ & $74.7 \pm 6.1$ \\
7 & $0.94 \pm 0.16$ & $69.2 \pm 10.3$ & $74.2 \pm 6.1$ \\
8 & $0.95 \pm 0.17$ & $69.7 \pm 10.4$ & $74.1 \pm 6.6$ \\
9 & $0.95 \pm 0.21$ & $68.2 \pm 10.3$ & $73.3 \pm 7.9$ \\
10 & $0.95 \pm 0.16$ & $69.2 \pm 10.2$ & $73.6 \pm 7.8$ \\
11 & $0.95 \pm 0.18$ & $68.5 \pm 11.3$ & $73.0 \pm 8.1$ \\
12 & $0.95 \pm 0.16$ & $70.1 \pm 11.5$ & $74.4 \pm 7.2$ \\
13 & $0.93 \pm 0.16$ & $68.2 \pm 11.0$ & $73.5 \pm 7.5$ \\
14 & $0.94 \pm 0.16$ & $68.6 \pm 11.7$ & $73.8 \pm 7.9$
\end{tabular}

(continued) 
Table 2. Continued

\begin{tabular}{lccc}
\hline Hour & cTOI/pTOI-ratios & cTOI $(\%)$ & pTOI (\%) \\
\hline 15 & $0.95 \pm 0.18$ & $68.2 \pm 10.8$ & $72.9 \pm 8.3$ \\
16 & $0.99 \pm 0.17$ & $71.2 \pm 9.6$ & $73.0 \pm 7.7$ \\
17 & $0.95 \pm 0.17$ & $69.9 \pm 11.1$ & $73.8 \pm 7.0$ \\
18 & $0.95 \pm 0.16$ & $69.6 \pm 11.0$ & $73.4 \pm 6.7$ \\
19 & $0.98 \pm 0.16$ & $70.7 \pm 10.2$ & $72.5 \pm 7.6$ \\
20 & $0.98 \pm 0.14$ & $70.5 \pm 10.1$ & $72.6 \pm 7.4$ \\
21 & $0.98 \pm 0.18$ & $70.1 \pm 10.5$ & $72.2 \pm 8.6$ \\
22 & $1.00 \pm 0.17$ & $71.1 \pm 8.9$ & $71.7 \pm 7.3$ \\
23 & $1.00 \pm 0.15$ & $71.7 \pm 10.0$ & $72.3 \pm 5.8$ \\
24 & $0.99 \pm 0.19$ & $70.1 \pm 10.3$ & $71.9 \pm 7.1$ \\
\hline
\end{tabular}

Table 3. Mean monitoring parameters ( $\mathrm{HR}, \mathrm{SpO}_{2}, \mathrm{MABP}$, rectal and peripheral temperature) of preterm neonates during the 24 -h measuring period. Data are presented as mean $\pm \mathrm{SD}$

\begin{tabular}{lccccc}
\hline Hour & $\begin{array}{c}\mathrm{HR} \\
(\mathrm{bpm})\end{array}$ & $\begin{array}{c}\mathrm{SpO}_{2} \\
(\%)\end{array}$ & $\begin{array}{c}\mathrm{MABP} \\
(\mathrm{mmHg})\end{array}$ & $\begin{array}{c}\text { Rectal temperature } \\
\left({ }^{\circ} \mathrm{C}\right)\end{array}$ & $\begin{array}{c}\text { Peripheral temperature } \\
\left({ }^{\circ} \mathrm{C}\right)\end{array}$ \\
\hline 1 & $148 \pm 13$ & $96 \pm 3$ & $38.9 \pm 6.1$ & $37.0 \pm 0.4$ & $37.1 \pm 0.4$ \\
2 & $147 \pm 13$ & $96 \pm 2$ & $39.6 \pm 6.4$ & $37.1 \pm 0.3$ & $37.1 \pm 0.3$ \\
3 & $145 \pm 14$ & $96 \pm 2$ & $39.8 \pm 6.6$ & $37.1 \pm 0.3$ & $37.2 \pm 0.3$ \\
4 & $143 \pm 13$ & $96 \pm 2$ & $40.8 \pm 7.3$ & $37.0 \pm 0.3$ & $37.1 \pm 0.3$ \\
5 & $143 \pm 12$ & $96 \pm 3$ & $41.8 \pm 6.2$ & $37.3 \pm 0.3$ & $37.2 \pm 0.3$ \\
6 & $142 \pm 13$ & $96 \pm 3$ & $42.5 \pm 7.5$ & $37.1 \pm 0.4$ & $37.1 \pm 0.5$ \\
7 & $141 \pm 12$ & $96 \pm 2$ & $43.4 \pm 6.8$ & $37.1 \pm 0.3$ & $37.0 \pm 0.7$ \\
8 & $141 \pm 11$ & $96 \pm 3$ & $43.6 \pm 7.7$ & $37.1 \pm 0.3$ & $37.2 \pm 0.3$ \\
9 & $143 \pm 12$ & $96 \pm 3$ & $44.2 \pm 6.8$ & $37.1 \pm 0.3$ & $37.1 \pm 0.4$ \\
10 & $144 \pm 12$ & $96 \pm 3$ & $44.2 \pm 7.3$ & $37.0 \pm 0.3$ & $37.0 \pm 0.4$ \\
11 & $142 \pm 12$ & $96 \pm 3$ & $44.8 \pm 7.5$ & $37.2 \pm 0.4$ & $37.2 \pm 0.4$ \\
12 & $141 \pm 12$ & $96 \pm 3$ & $44.8 \pm 7.2$ & $37.0 \pm 0.3$ & $37.1 \pm 0.3$ \\
13 & $140 \pm 12$ & $96 \pm 3$ & $44.0 \pm 6.0$ & $37.1 \pm 0.3$ & $37.1 \pm 0.2$ \\
14 & $142 \pm 12$ & $96 \pm 3$ & $44.8 \pm 7.5$ & $37.2 \pm 0.4$ & $37.0 \pm 0.3$ \\
15 & $141 \pm 12$ & $96 \pm 3$ & $45.4 \pm 6.4$ & $37.1 \pm 0.3$ & $37.1 \pm 0.2$ \\
16 & $140 \pm 12$ & $96 \pm 2$ & $44.6 \pm 6.6$ & $37.1 \pm 0.2$ & $37.2 \pm 0.3$ \\
17 & $141 \pm 12$ & $96 \pm 3$ & $45.2 \pm 7.7$ & $37.1 \pm 0.2$ & $37.1 \pm 0.4$ \\
18 & $141 \pm 12$ & $96 \pm 2$ & $45.1 \pm 7.2$ & $37.1 \pm 0.4$ & $37.0 \pm 0.3$ \\
19 & $141 \pm 10$ & $96 \pm 3$ & $44.4 \pm 6.6$ & $37.1 \pm 0.4$ & $37.1 \pm 0.3$ \\
20 & $142 \pm 11$ & $96 \pm 3$ & $44.8 \pm 6.7$ & $37.1 \pm 0.3$ & $37.1 \pm 0.3$ \\
21 & $141 \pm 11$ & $96 \pm 2$ & $44.5 \pm 6.1$ & $37.2 \pm 0.3$ & $36.9 \pm 0.4$ \\
22 & $141 \pm 11$ & $96 \pm 3$ & $44.0 \pm 6.3$ & $37.1 \pm 0.2$ & $37.1 \pm 0.3$ \\
23 & $141 \pm 11$ & $96 \pm 3$ & $45.3 \pm 7.5$ & $37.0 \pm 0.2$ & $37.1 \pm 0.2$ \\
24 & $142 \pm 12$ & $96 \pm 3$ & $43.3 \pm 7.2$ & $37.1 \pm 0.2$ &
\end{tabular}

excluded due to invalid NIRS measurements $(n=9)$ or withdrawal of informed consent by the parents $(n=1)$. Out of the remaining 98 preterm neonates another $11(11.2 \%)$ had to be excluded from the present analyses due to early signs of infection/inflammation in combination 

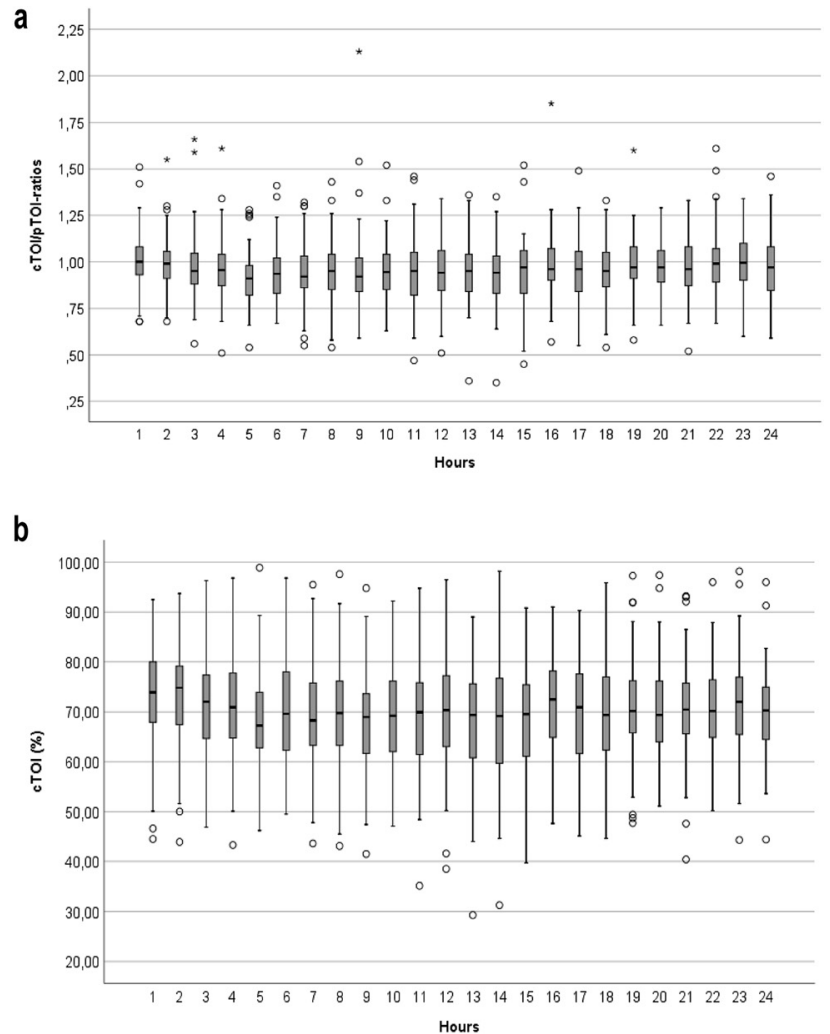

C

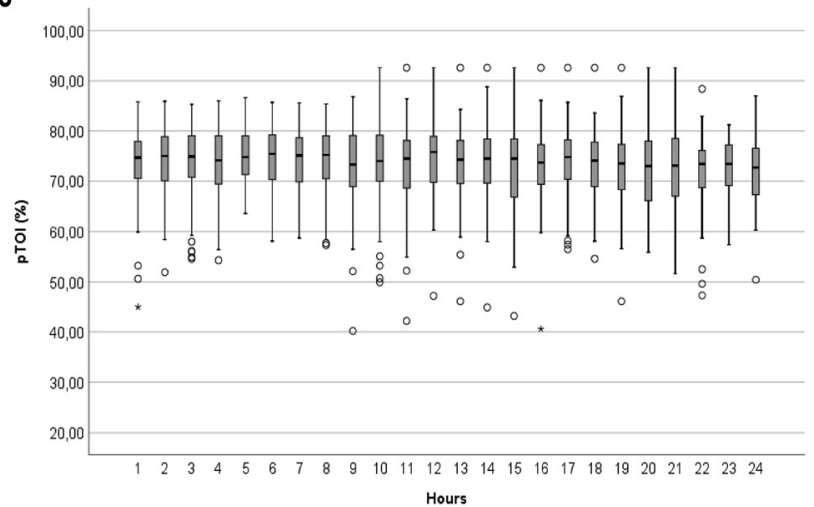

Fig. 2 a. cTOI/pTOI-ratios of preterm neonates during the 24-h measuring period. b. cTOI values of preterm neonates during the 24 - $\mathrm{h}$ measuring period. c. pTOI values of preterm neonates during the 24 -h measuring period

with $(n=2)$ /without positive blood cultures $(n=9)$ (Fig. 1$)$. Of the remaining 87 neonates, one had positive blood culture without clinical/laboratory signs of inflammation/infection, thus, most likely due to contamination, and was therefore retained in the study for further analysis. 
These remaining 87 preterm neonates $(88.8 \%)$ were admitted to the NICU due to the following conditions: 38 neonates (43.7\%) suffered from idiopathic respiratory distress syndrome, 28 neonates (32.2\%) were premature and 21 (24.1\%) showed signs of transient tachypnoea of the newborn. The demographic and clinical data of the neonates included are presented in Table 1. Twenty-eight neonates (32.2\%) received non-invasive respiratory support and four neonates $(4.6 \%)$ were mechanically ventilated. Surfactant was administered in nine neonates $(10.3 \%)$. None of the neonates was resuscitated with chest compressions or received catecholamines. cTOI/pTOI-ratios as well as cTOI and pTOI values of every hour during the 24$\mathrm{h}$ measuring period are presented in Table 2 . The mean value over the 24 -h measuring period for the cTOI/pTOI-ratio was $0.96 \pm 0.02$, for cTOI $70.1 \pm 1.4 \%$ and for pTOI $73.4 \pm 0.9 \%$.

Routine monitoring parameter values $\left(\mathrm{HR}, \mathrm{SpO}_{2}, \mathrm{MABP}\right.$, rectal and peripheral temperature) of every hour during the 24 -h measuring period are presented in Table 3 . Mean values over the 24-h measuring period were: $\mathrm{HR}, 142 \pm 2 \mathrm{bpm}$; $\mathrm{SpO}_{2}, 96 \pm 0 \%$; MABP, $43.5 \pm 1.9 \mathrm{mmHg}$; and rectal and peripheral temperature, $37.1 \pm 0.1{ }^{\circ} \mathrm{C}$ each. cTOI/pTOI-ratios and cTOI values showed significantly lower values from hour 5 to 15 compared to the first hours after birth, followed by a slight increase during hour 16-24 (Tables 2 and 4, Fig. 2a and b). HR showed a significant decrease over time, whereas MABP values increased significantly (Tables 3 and 5).

Table 4. $P$-values of mixed model ANOVA comparing NIRS parameters over the 24-h measuring period to the first hour of measurements $\left({ }^{\star} P<0.05\right)$

\begin{tabular}{|c|c|c|c|}
\hline Hour & cTOI/pTOI-ratio & cTOI (\%) & $\begin{array}{c}\text { pTOI } \\
(\%)\end{array}$ \\
\hline 2 & 0.322 & 0.492 & 0.469 \\
\hline 3 & 0.081 & 0.142 & 0.210 \\
\hline 4 & 0.114 & 0.053 & 0.842 \\
\hline 5 & $0.003^{*}$ & $0.011^{*}$ & 0.075 \\
\hline 6 & $0.035^{\star}$ & 0.066 & 0.253 \\
\hline 7 & $0.010^{*}$ & $0.010^{*}$ & 0.338 \\
\hline 8 & $0.021^{\star}$ & $0.011^{\star}$ & 0.610 \\
\hline 9 & $0.027^{\star}$ & $0.001^{*}$ & 0.765 \\
\hline 10 & $0.020^{*}$ & $0.003^{*}$ & 0.989 \\
\hline 11 & $0.015^{\star}$ & $0.002^{*}$ & 0.905 \\
\hline 12 & $0.027^{\star}$ & $0.030^{*}$ & 0.522 \\
\hline 13 & $0.007^{\star}$ & $0.003^{*}$ & 0.775 \\
\hline 14 & $0.009^{*}$ & $0.003^{*}$ & 0.919 \\
\hline 15 & $0.038^{\star}$ & $0.006^{*}$ & 0.792 \\
\hline 16 & 0.266 & 0.072 & 0.676 \\
\hline 17 & 0.065 & $0.023^{*}$ & 0.994 \\
\hline 18 & 0.057 & $0.026^{*}$ & 0.975 \\
\hline 19 & 0.220 & 0.064 & 0.664 \\
\hline 20 & 0.389 & 0.088 & 0.305 \\
\hline 21 & 0.407 & $0.041^{*}$ & 0.178 \\
\hline 22 & 0.969 & 0.175 & 0.065 \\
\hline 23 & 0.960 & 0.265 & 0.147 \\
\hline 24 & 0.914 & 0.472 & 0.296 \\
\hline
\end{tabular}


Table 5. $P$-values of mixed model ANOVA comparing routine monitoring parameters over the 24-h measuring period to the first hour of measurements $\left({ }^{\star} P<0.05\right)$

\begin{tabular}{|c|c|c|c|c|c|}
\hline Hour & $\begin{array}{c}\mathrm{HR} \\
(\mathrm{bpm})\end{array}$ & $\begin{array}{c}\mathrm{SpO}_{2} \\
(\%)\end{array}$ & $\begin{array}{l}\text { MABP } \\
(\mathrm{mmHg})\end{array}$ & $\begin{array}{c}\text { Rectal temperature } \\
\left({ }^{\circ} \mathrm{C}\right)\end{array}$ & $\begin{array}{c}\text { Peripheral temperature } \\
\left({ }^{\circ} \mathrm{C}\right)\end{array}$ \\
\hline 2 & 0.120 & 0.810 & 0.456 & 0.383 & 0.688 \\
\hline 3 & $0.009^{\star}$ & 0.842 & 0.397 & 0.140 & 0.707 \\
\hline 4 & $0.001^{*}$ & 0.315 & 0.113 & 0.868 & 0.606 \\
\hline 5 & $0.001^{\star}$ & 0.526 & $0.014^{\star}$ & $0.020^{\star}$ & 0.959 \\
\hline 6 & $0.000^{\star}$ & 0.845 & $0.004^{\star}$ & 0.240 & 0.477 \\
\hline 7 & $0.000^{\star}$ & 0.416 & $\mathbf{0 . 0 0 0}^{\star}$ & 0.596 & 0.338 \\
\hline 8 & $0.000^{\star}$ & 0.491 & $0.000^{*}$ & 0.287 & 0.465 \\
\hline 9 & $0^{0.008^{*}}$ & 0.568 & $0.000^{\star}$ & 0.636 & 0.893 \\
\hline 10 & $0.016^{\star}$ & 0.997 & $\mathbf{0 . 0 0 0}^{\star}$ & 0.737 & 0.463 \\
\hline 11 & $0.001^{*}$ & 0.661 & $0.000^{*}$ & 0.124 & 0.709 \\
\hline 12 & $0.000^{\star}$ & 0.993 & $0.000^{*}$ & 0.798 & 0.663 \\
\hline 13 & $0.000^{*}$ & 0.873 & $0.000^{*}$ & 0.618 & 0.644 \\
\hline 14 & $0.001^{\star}$ & 0.945 & $\mathbf{0 . 0 0 0}^{\star}$ & 0.086 & 0.458 \\
\hline 15 & $0.000^{\star}$ & 0.746 & $0.000^{\star}$ & 0.451 & 0.696 \\
\hline 16 & $0.000^{*}$ & 0.708 & $0.000^{*}$ & 0.591 & 0.367 \\
\hline 17 & $0.000^{*}$ & 0.183 & $0.000^{*}$ & 0.219 & 0.801 \\
\hline 18 & $0.000^{*}$ & 0.675 & $0.000^{*}$ & 0.261 & 0.854 \\
\hline 19 & $0.000^{\star}$ & 0.309 & $0.000^{\star}$ & 0.435 & 0.730 \\
\hline 20 & $0.001^{\star}$ & 0.966 & $0.000^{*}$ & 0.311 & 0.524 \\
\hline 21 & $0.001^{\star}$ & 0.474 & $\mathbf{0 . 0 0 0}^{*}$ & 0.070 & 0.595 \\
\hline 22 & $\mathbf{0 . 0 0 0}^{*}$ & 0.549 & $\mathbf{0 . 0 0 0}^{*}$ & 0.654 & 0.178 \\
\hline 23 & $\mathbf{0 . 0 0 0}^{*}$ & 0.796 & $\mathbf{0 . 0 0 0}^{*}$ & 0.911 & 0.530 \\
\hline 24 & $0.001^{\star}$ & 0.626 & $0.000^{\star}$ & 0.362 & 0.909 \\
\hline
\end{tabular}

pTOI (Tables 2 and 4, Fig. 2c), $\mathrm{SpO}_{2}$, as well as rectal and peripheral temperature (Tables 3 and 5) showed no change over time.

No severe adverse reactions were observed during NIRS measurements. In regard to cerebral injuries, four neonates (4.6\%) developed unilateral intraventricular haemorrhage grade I and one neonate $(1.1 \%)$ suffered from bilateral periventricular leukomalacia grade I. None of the included neonates died.

\section{DISCUSSION}

To our knowledge, we have introduced cTOI/pTOI-ratios for every hour during the first day after birth for the first time. In our cohort of healthy preterm neonates, cTOI/pTOI-ratios showed significantly lower values from hour 5 to 15 compared to the first hours after birth. The mean cTOI/pTOI-ratio over the 24-h measuring period was $0.96 \pm 0.02$, as pTOI values were similar to cTOI values. At first sight this seems to be in contrast to the findings of Grossauer et al. [12], who described higher cTOI values within the first eight weeks of life compared to pTOI values $(70.1 \pm 6.7 \%$ vs. $62.1 \pm 5.7 \%)$. Consequently, the mean cTOI/pTOI-ratio in the present study was lower compared to the cTOI/pTOI-ratio published by Grossauer et al. [12] 
$(1.14 \pm 0.14)$. However, the differences between the two studies can be explained by the different postnatal age of included patients and the physiological decrease of pTOI and increase of cTOI during the first days after birth [15,22]. Schmitz et al. [23] described higher peripheral muscle $\mathrm{rSO}_{2}(83.5 \pm 0.9)$ values compared to cerebral $\mathrm{rSO}_{2}(78.5 \pm 1.2 \%)$ during the first $24 \mathrm{~h}$ after birth in preterm and term neonates. When calculating a $\mathrm{crSO}_{2} / \mathrm{prSO}_{2}$ ratio for the first $24 \mathrm{~h}$ after birth, the result of 0.94 is similar to our findings.

In the present study cTOI showed the lowest values from hour 5 to 15 during the first day after birth, while pTOI remained stable, resulting in a decrease of cTOI/pTOI-ratio during this time period. Again, these changes seem to be in contrast to recent findings. Pichler et al. [15] described a decrease of pTOI in healthy term neonates from $67 \%$ to $61 \%$ during the first week of life. On the other hand, cTOI increases in premature infants over the first three days of life from $57 \%$ to $76.1 \%$ [16]. However, we speculate that differences to our results can be explained by different time periods and different sampling periods. Sirc et al. [24] as well as Takami et al. [25] also described a decrease in cTOI during the first $12 \mathrm{~h}$ after birth, which is in accordance with our results. These changes in cerebral oxygen saturation were interpreted as a consequence of cerebral and cardiac haemodynamic changes during this transitional period.

During the first hours after birth we observed, on the one hand, a reduction of HR that might be associated with a reduction of cardiac output. On the other hand, we observed an increase in MABP that is most probably due to an increase in vascular resistance. Both mechanisms might also play a role in cerebral haemodynamics with a reduction of cerebral perfusion causing the observed decrease in cTOI. Furthermore, cTOI is also influenced by changes in oxygen consumption that might have contributed to the present findings. However, exact underlying mechanism and influence of cerebral autoregulation on the observed changes, especially on cTOI, during the first day after birth cannot be ruled out completely with the present data.

Taking present and recent findings together, cTOI seems to decrease at some time points on the first day after birth, whereas pTOI does not change significantly, whereas thereafter cTOI increases and pTOI starts to decrease, resulting in different cTOI/pTOI-ratios depending on postnatal age.

Mean cTOI values of our study are within the target ranges for cerebral NIRS measurements of 55-85\%, published by Hytell-Sorensen et al. [26]. Nevertheless these target ranges were published using the INVOS 5100c (Covidien, Boulder, CO) and differences to other NIRS devices have to be taken into account.

Concerning routine monitoring parameters over the 24-h measuring period, all measured values were in the normal ranges for preterm neonates. None of the neonates had severe arterial hypotension or needed treatment with catecholamines. A possible explanation may be that mainly moderate or late preterm neonates with compensated cardio-circulatory conditions were included.

Additionally, we also investigated courses of values of routine monitoring parameters in these healthy preterm neonates during the first day after birth. HR values decreased significantly over the 24-h measuring period compared to the first hour of measurements, whereas MABP, as reported in other studies [27], increased significantly from hours five to 24. Interestingly, $\mathrm{SpO}_{2}$ did not show any significant difference over time. O'Brien et al. [28] investigated $\mathrm{SpO}_{2}$ in healthy term infants throughout the first $24 \mathrm{~h}$ of life, reporting baseline $\mathrm{SpO}_{2}$ values of $98.3 \%$ with stable values until hour 20 and thereafter a significant decrease until the postnatal age of 24 $\mathrm{h}$. Rectal and peripheral body temperatures always remained in normothermic range over the study period. 


\section{LIMITATIONS}

In the present study mainly moderate to late preterm neonates were included, thus we cannot comment on potential differences of cTOI/pTOI-ratios in extremely preterm neonates. We did not include extremely preterm neonates, since the application of the peripheral NIRS sensors in these vulnerable patients is technically challenging and often not feasible.

There is a wide range of cTOI and pTOI that can be explained by supposing that in some neonates there might have been some artefacts due to loose of optical contact, which was not recognized during the 24-h measurement period. However, as the Figures demonstrate, the extreme values can be classified as outliers in both directions, suggesting no influence on mean values.

\section{CONCLUSION}

We are the first to report on cTOI/pTOI-ratios for healthy preterm neonates over a 24-h period after birth, which show significantly lower values from hour 5 to 15 compared to the first hours after birth. Continuous, simultaneous NIRS measurements of cerebral and peripheral muscle oxygenation may have the potential to detect early and still mild stages of cardio-circulatory disturbances, allowing for risk stratification of preterm neonates who are at risk of sepsis. Since changes in the cTOI/pTOI-ratio might be an early marker of centralization, it may be an even better predictive marker for disturbances of the peripheral (micro-)circulation than single NIRS or routine monitoring parameters. Further studies, especially in neonates with manifest infection/sepsis, will therefore be of great interest.

\section{ACKNOWLEDGEMENTS}

We would like to express our gratitude to the parents for putting us in position to investigate their infants, as well as all the midwives, nurses, and physicians involved in the treatment of these neonates. Furthermore we want to thank the study nurse Evelyn Ziehenberger for her assistance in completing this study, and the statistician PD Mag. Dr. Alexander Avian for assistance with the statistical analysis.

\section{REFERENCES}

1. Bateman RM, Sharpe MD, Ellis CG. Bench-to-bedside review: microvascular dysfunction in sepsis-hemodynamics, oxygen transport, and nitric oxide. Crit Care 2003; 7: 359-73.

2. Spronk PE, Zandstra DF, Ince C. Bench-to-bedside review: sepsis is a disease of the microcirculation. Crit Care 2004; 8: 462-8.

3. Nanas S, Gerovasili V, Renieris P, Angelopoulos E, Poriazi M, Kritikos K, et al. Non-invasive assessment of the microcirculation in critically ill patients. Anaesth Intensive Care 2009; 37: 733-9.

4. Ince C. The microcirculation is the motor of sepsis. Crit Care 2005; 9(Suppl 4): S13-9. 
5. Dullenkopf A, Kolarova A, Schulz G, Frey B, Baenziger O, Weiss M. Reproducibility of cerebral oxygenation measurement in neonates and infants in the clinical setting using the NIRO 300 oximeter. Pediatr Crit Care Med 2005; 6: 344-7.

6. Greisen G. Is near-infrared spectroscopy living up to its promises? Semin Fetal Neonatal Med 2006; 11: 498-502.

7. Höller N, Urlesberger B, Mileder L, Baik N, Schwaberger B, Pichler G. Peripheral muscle near-infrared spectroscopy in neonates: ready for clinical use? A systematic qualitative review of the literature. Neonatology 2015; 108: 233-45.

8. Nicklin SE, Hassan IAA, Wickramasinghe YA, Spencer SA. The light still shines, but not that brightly? The current status of perinatal near infrared spectroscopy. Arch Dis Child Fetal Neonatal Ed 2003; 88: F263-8.

9. Pocivalnik M, Pichler G, Zotter H, Tax N, Müller W, Urlesberger B. Regional tissue oxygen saturation: comparability and reproducibility of different devices. J Biomed Opt 2011; 16: 057004.

10. Sorensen LC, Leung TS, Greisen G. Comparison of cerebral oxygen saturation in premature infants by nearinfrared spatially resolved spectroscopy: observations on probe-dependent bias. J Biomed Opt 2008; 13: 064013.

11. Wolfberg AJ, du Plessis AJ. Near-infrared spectroscopy in the fetus and neonate. Clin Perinatol 2006; 33: 707-28.

12. Grossauer K, Pichler G, Schmolzer G, Zotter H, Mueller W, Urlesberger B. Comparison of peripheral and cerebral tissue oxygenation index in neonates. Arch Dis Child Fetal Neonatal Ed 2009; 94: F156.

13. Schwaberger B, Pichler G, Binder C, Avian A, Pocivalnik M, Urlesberger B. Even mild respiratory distress alters tissue oxygenation significantly in preterm infants during neonatal transition. Physiol Meas 2014; 35: 2085-99.

14. Urlesberger B, Grossauer K, Pocivalnik M, Avian A, Müller W, Pichler G. Regional oxygen saturation of the brain and peripheral tissue during birth transition of term infants. J Pediatr 2010; 157: 740-4.

15. Pichler G, Grossauer K, Klaritsch P, Kutschera J, Zotter H, Müller W, et al. Peripheral oxygenation in term neonates. Arch Dis Child Fetal Neonatal Ed 2007; 92: F51-2.

16. Naulaers G, Morren G, Van Huffel S, Casaer P, Devlieger H. Measurement of tissue oxygenation index during the first three days in premature born infants. Adv Exp Med Biol 2003; 510: 379-83.

17. Pichler G, Höller N, Baik-Schneditz N, Schwaberger B, Mileder L, Stadler J, et al. Avoiding Arterial Hypotension in Preterm Neonates (AHIP) - a single center randomised controlled study investigating simultaneous near infrared spectroscopy measurements of cerebral and peripheral regional tissue oxygenation and dedicated interventions. Front Pediatr 2018; 6: 15.

18. Messer J, Eyer D, Donato L, Gallati H, Matis J, Simeoni U. Evaluation of interleukin-6 and soluble receptors of tumor necrosis factor for early diagnosis of neonatal infection. J Pediatr 1996; 129: 574-80.

19. Goldstein B, Giroir B, Randolph A, International Consensus Conference on Pediatric Sepsis. International pediatric sepsis consensus conference: definitions for sepsis and organ dysfunction in pediatrics. Pediatr Crit Care Med 2005; 6: 2-8.

20. Mathers NJ, Pohlandt F. Diagnostic audit of C-reactive protein in neonatal infection. Eur J Pediatr 1987; 146: 147-51.

21. Pichler G, Pocivalnik M, Riedl R, Pichler-Stachl E, Zotter H, Müller W, et al. C reactive protein: impact on peripheral tissue oxygenation and perfusion in neonates. Arch Dis Child Fetal Neonatal Ed 2012; 97: F444-8.

22. Pichler G, Pocivalnik M, Riedl R, Pichler-Stachl E, Morris N, Zotter H, et al. 'Multi-associations': predisposed to misinterpretation of peripheral tissue oxygenation and circulation in neonates. Physiol Meas 2011; 32: $1025-34$. 
23. Schmitz J, Pichler G, Schwaberger B, Urlesberger B, Baik N, Binder C. Feasibility of long-term cerebral and peripheral regional tissue oxygen saturation measurements. Physiol Meas 2014; 35: 1349-55.

24. Sirc J, Dempsey EM, Miletin J. Cerebral tissue oxygenation index, cardiac output and superior vena cava flow in infants with birth weight less than 1250 grams in the first 48 hours of life. Early Hum Dev 2013; 89: 449-52.

25. Takami T, Sunohara D, Kondo A, Mizukaki N, Suganami Y, Takei Y, et al. Changes in cerebral perfusion in extremely low birth weight infants during the first 72 hours after birth. Pediatr Res 2010; 68: 435-9.

26. Hyttel-Sorensen S, Pellicer A, Alderliesten T, Austin T, van Bel F, Benders M, et al. Cerebral near infrared spectroscopy oximetry in extremely preterm infants: phase II randomised clinical trial. BMJ 2015; 350 : g7635.

27. Cordero L, Timan CJ, Waters HH, Sachs LA. Mean arterial pressures during the first 24 hours of life in $\leq 600$ gram birth weight infants. J Perinatol 2002; 22: 348-53.

28. O'Brien LM, Stebbens VA, Poets CF, Heycock EG, Southall DP. Oxygen saturation during the first 24 hours of life. Arch Dis Child Fetal Neonatal Ed 2000; 83: F35-8. 